\title{
The problem of exogenous subclinical hyperthyroidism
}

\author{
Menelaos L. Batrinos
}

Emeritus Professor of Endocrinology, Athens University Medical School, Athens, Greece

\begin{abstract}
Over the past two decades a plethora of publications and clinical practice data have established that subclinical thyroid dysfunction is a common condition occurring much more frequently than the overt expression of thyroid disease. Subclinical hypothyroidism is considered to be the most common of these entities. However, far more common and relatively less studied is exogenous sublinical hyperthyroidism (SubHyper) caused by L-thyroxine (T4) administration to thyroidectomized or hypothyroid patients or patients with simple or nodular goiter. Despite iodination, simple goiter is still prevalent and single or multiple nodules are now detected by ultrasound screening in $\mathbf{2 5 - 3 0 \%}$ of adults, who are accordingly frequently given long-term T4 treatment. Approximately half of European Endocrinologists administer T4 permanently to patients with the above entities with the aim of suppressing TSH levels. Furthermore, in the USA the Colorado Study demonstrated that $40 \%$ of patients receiving thyroid hormones had suppressed TSH levels (less than $0.3 \mathrm{mIU} / \mathrm{L}$ ). These facts render exogenous SubHyper an everyday problem for the endocrinologist. Exogenous SubHyper differs in many aspects from endogenous, its principal difference being that it is an iatrogenic thyroid disorder induced by the endocrinologist. The management of exogenous SubHyper relies on appropriate adjustment of $\mathbf{T} 4$ dosage taking into consideration a) individual requirements in $\mathbf{T} 4$, sex, age and the presence of cardiovascular disease or other co-morbidity, b) the recognition that small changes in serum FT4 have a logarithmic effect on TSH, c) the variability of FT4TSH interactions between individuals, d) the instability of T4 preparations and its bioavailability, and e) the values of serum FT4 and FT3 that accompany a suppressed TSH. This last parameter is of importance since it is the free thyroid hormones values in the serum that generate and reflect the thyroid metabolic state of the organism rather than the degree of TSH suppression.
\end{abstract}

Key words: Exogenous hyperthyroidism, Hyperthyroidism, Subclinical hyperthyroidism

Over the past two decades a plethora of publications and clinical practice data have established that

\footnotetext{
Address correspondence and requests for reprints to: Professor Menelaos L. Batrinos, 8 Evripidou Str.,

Politeia 145 63, Greece, Tel. +30 2106204041 ,

E-mail: bithrini@ath.forthnet.gr

Received 01-02-06, Revised 10-03-06, Accepted 20-03-06
}

subclinical thyroid dysfunction is a common condition occurring much more frequently than the overt expression of thyroid disease. However, despite extensive literature on the subject, controversial issues remain regarding screening, evaluation of clinical importance and management of patients with this disorder defined as subclinical hypo- or hyperthy- 
roidism. Since no definite clinical signs are present, the diagnosis of subclinical thyroid dysfunction relies on reference range of thyrotropin values derived from a study of "disease free", ethnically diverse groups of 13,344 individuals. ${ }^{1}$ In this study TSH concentrations of the $2.5^{\text {th }}$ and $97.5^{\text {th }}$ percentiles were found to be 0.45 and $4.12 \mathrm{mIU} / \mathrm{L}$, respectively, and were considered as the lower and upper normal limits of the general population. Based on these results, a consensus committee of experts ${ }^{2}$ defined subclinical hypothyroidism as the condition presenting serum TSH concentration above $4.5 \mathrm{mIU} / \mathrm{L}$ and normal thyroxine (T4) and triodothyronine (T3) levels. It was, however, mentioned that certain authors suggest that the upper limit of normal for TSH concentration should be $2.5 \mathrm{mIU} / \mathrm{L} .^{2-5}$ The subclinical form of hyperthyroidism was characterized as a serum concentration of $0.45 \mathrm{mIU} / \mathrm{L}$ or less, accompanied by normal $\mathrm{T} 4$ and $\mathrm{T} 3$ values.

Subclinical hypothyroidism is considered to be the most common thyroid dysfunction, its prevalence being about $4-9.5 \%$, this increasing to more than $20 \%$ in old age, especially in women. ${ }^{6-8}$ In the U.S., based on epidemiological data and the 2000 U.S. concensus, it was estimated that approximately 9.6 million women and 4.4 million men have subclinical hypothyroidism. ${ }^{9}$ Subclinical hyperthyroidism is much less common in the disease-free population not taking thyroxine, being about $2 \%$ and rising to $5.5 \%$ in males and $6.5 \%$ in females past the age of 60 years.

However, far more common than the above-mentioned dysfunctions and relatively less studied is exogenous subclinical hyperthyroidism (SubHyper) caused by thyroxine administration for various thyroid nosologies. The prevalence of this condition has not been defined but can be deduced from indirect evidence. Patients thyroidectomized for thyroid cancer are given high doses of thyroxine and hypothyroid patients receiving substitution therapy with thyroxine are potential candidates for exogenous subclinical hyperthyroidism. The bulk of patients, however, receiving thyroxine are those with simple or nodular goiter. Simple goiter, despite iodination, is still prevalent and the incidence of multi-nodular goiter is high. In the Whickham survey of 2779 subjects, $5.3 \%$ of adult women and $0.8 \%$ of men had clinically palpable thyroid nodules. ${ }^{6}$ With ultrasound screening, single or multiple nodules are found in 25 to $30 \%$ of adults and up to $60 \%$ in women over 60 years of age, most of whom are given long-term thyroxine treatment. ${ }^{10,11}$ An ultrasonography screening of 96,278 unselected employees in Germany demonstrated the presence of goiter and/or nodules $>0.5 \mathrm{~cm}$ in $34.2 \%$ of women and $32 \%$ of men. ${ }^{12}$ In Europe, $50 \%$ of the members of the European Thyroid Association who replied to a questionnaire stated that their treatment recommendations to the patients were to take thyroxine permanently, aiming at a suppressed TSH level. ${ }^{13}$

The above categories of patients constitute a huge body of people receiving thyroxine in doses ranging from $100 \mu \mathrm{g}$ to $250 \mu \mathrm{g}$ daily, which, in a considerable proportion of them, exerts a suppressive effect on TSH. This is documented by the NHANES III survey in which $18.3 \%$ of the 820 people taking thyroid medication had biochemical evidence of hyperthyroidism (7.3\% clinical, $10.9 \%$ subclinical), as well as the Colorado Study which found that $40 \%$ of patients receiving thyroid hormones had abnormal TSH levels (less than $0.3 \mathrm{mIU} / \mathrm{L}){ }^{1,7}$

Because of its high incidence, exogenous SubHyper presents an everyday problem to clinical endocrinologists and, because it differs in many respects from endogenous SubHyper, its characteristics need to be delineated. Firstly, its etiology is comprehended whereas in the endogenous form a variety of causes have to be investigated such as an autonomous adenoma, hyperfunctioning multiple nodules, relapse or initial stages of clinical hyperthyroidism, subacute thyroiditis, the use of drugs as cortisone, amiodarone, iodine, dopamine and even certain non thyroidal illnesses. The duration of exogenous SubHyper can more easily be calculated from the medical history of the patient, whereas in the endogenous variety this significant parameter for the impact of thyroid dysfunction on the organism is unknown. Exogenous SubHyper does not carry the risk of progressing to overt hyperthyroidism as has been reported to happen yearly in 5\% of patients with endogenous SubHyper. Furthermore, euthyroidism is easily achieved in exogenous SubHyper, necessitating only delicate manipulations and individualization of thyroxine dosage without resort to drug 
or other forms of therapy. What is specific to exogenous SubHyper is that this thyroid dysfunction is iatrogenic and often produced by the very same clinician who makes its diagnosis.

With these characteristics the problem of exogenous SubHyper is focused on the following issues: 1. the risks of suppressive doses of thyroxine; 2 . the need to categorize the patients; 3 . the veritable metabolic significance and implications of a low TSH value; and 4 . the therapeutic strategy.

1. The risks of subclinical thyroid dysfunctions, including exogenous SubHyper, were recently explored by a committee of the American Thyroid Association (ATA), the American Association of Clinical Endocrinologists (AACE) and the American Endocrine Society with the aim of formulating guidelines for the clinicians. ${ }^{2}$ The members of the Committee reviewed all English language research articles (N 195) and evaluated the strength of the evidence regarding the association of subclinical thyroid states and abnormal clinical outcomes. The panel, following the approach taken by many investigators, classified patients with SubHyper into two categories: those with serum TSH between 0.4 and $0.1 \mathrm{mIU} / \mathrm{L}$ and those with TSH values below $0.1 \mathrm{mIU} /$ $\mathrm{L}$, and analyzed evidence-based data regarding the consequences of SubHyper on the cardiovascular system, the skeleton and the neuropsychiatric and systemic symptoms. A related detailed summary of the data regarding subclinical thyroid dysfunction was also reported by Helfand from Oregon Health and Science University. ${ }^{14}$ Most of the papers analyzed concerned subclinical hypothyroidism and endogenous SubHyper with few notes on the exogenous form or the length of time of thyroid hormone administration. Nevertheless, the conclusions can, to a certain degree, be applied to exogenous SubHyper, taking into consideration the specific characteristics mentioned above. Two outstanding reviews of SubHyper with an extensive literature search appeared in 2005 and have been of great help to the present author in dealing with the problems of the exogenous SubHyper form. ${ }^{19,20}$

The cardiac dysfunction in patients with SubHyper reported in the literature includes increase in heart rate, arrhythmias, atrial fibrillation, increase of left ventricular mass and diastolic dysfunction. ${ }^{20}$ The panel of the ATA interpreted echocardiographic changes in endogenous SubHyper to "be small and of uncertain clinical importance". They, however, mentioned the study reporting an increase in cardiovascular mortality of up to threefold in patients older than 60 years with endogenous SubHyper and serum TSH lower than $0.5 \mathrm{mIU} / \mathrm{L} .{ }^{21}$ This study, however, was criticized in five letters to the Editor by various authors. ${ }^{22}$ Evidence for an increase of atrial fibrillation in patients with TSH 0.1 to 0.4 $\mathrm{mIU} / \mathrm{L}$ was considered by the ATA Committee to be limited, whereas the evidence was considered solid for those with TSH $<0.1 \mathrm{mIU} / \mathrm{L}$. A 10-year follow-up study of elderly patients with $\mathrm{TSH} \leq 0.1 \mathrm{mIU} /$ L disclosed a threefold higher incidence of atrial fibrillation compared with euthyroid subjects. ${ }^{23}$ In another retrospective study of 1138 subjects with TSH $<0.4 \mathrm{mIU} / \mathrm{L}$, the relative risk of atrial fibrillation was 5.3 compared with subjects with TSH value within the normal reference range. ${ }^{24}$ Biondi, based on her own research and literature data (2005), expressed an opinion differing from that of the ATA panel, stating that the most consistent cardiac abnormality in patients with exogenous and endogenous SubHyper is a significant increase in left ventricular mass. ${ }^{19}$ In a prospective placebo-controlled randomized trial, the T4 suppressive dose was reduced for 6 months in 33 patients with thyroid cancer. The results showed that, at baseline 4 patients had left ventricular hypertrophy and all patients had impaired diastolic function, which was at least partly reversed after restoration of euthyroidism..$^{25}$

The effect of SubHyper on the skeleton is extensively discussed in Biondi's et al. and Helfond's reviews, the overall conclusion being that it remains controversial whether SubHyper increases the risk of fractures. ${ }^{14,19,20}$ Two meta-analyses published in 1994 and 1996 found a reduction of bone mineral density during prolonged exogenous SubHyper among post-menopausal women but not in premenopausal women..$^{26,27}$ No significant effect on bone mineral content at femoral and spinal sites was found by Franklin et al in 44 pre- and postmenopausal women thyrectomized for thyroid cancer and on long-term (mean 7.9 years) high doses of thyroxine (mean $191 \pm 50 \mu \mathrm{g} /$ day) resulting in serum FT4 and 
FT3 at or slightly above the upper normal limit. ${ }^{28} \mathrm{~A}$ more recent prospective study of 686 women older than 65 years disclosed that women with TSH levels $<0.1 \mathrm{mIU} / \mathrm{L}$ had a threefold and fourfold increased risk for hip and vertebral fractures, respectively, compared to women with normal TSH values. In this study, however, no distinction was made between overt and SubHyper. ${ }^{29}$ In a nationwide survey of 11,776 patients with clinical hyperthyroidism, in which each patient was compared with 3 age -and gender-matched controls, fracture risk was statistically increased (1.26-2.29) only around the time of diagnosis but not after treatment. ${ }^{30}$

The existence of systemic symptoms or signs, even mild or few, are not, by definition, compatible with the diagnosis of SubHyper. Mild hyperthyroidism would be a more appropriate term in such cases which mainly concern endogenous SubHyper and may constitute an early stage of overt hyperthyroidism. In patients with exogenous SubHyper, Biondi et al reported signs and symptoms mimicking adrenergic overactivity which significantly improved after administration of b-blockers. ${ }^{31}$

2. The categorization of patients with exogenous SubHyper is important for their proper management. In young thyroidectomized patients with low risk differentiated carcinoma after several years of asymptomatic disease, the dose may be reduced to levels of TSH between $0.1-0.4 \mathrm{mIU} / \mathrm{L}$. In hypothyroid patients, replacement therapy can be adjusted to TSH concentrations up to $2.5 \mathrm{mIU} / \mathrm{L}$, a level considered by strict criteria to be the upper normal limit. In simple and nodular goiter, after whatever effect on the volume or nodules has been achieved in the first years of treatment, a trial with reduced maintainance dose can be tried accompanied by appropriate monitoring. A further categorization of all these patients should be made according to sex, age, medical history and physical condition in order to achieve the most appropriate therapeutic regimen. Women are more apt to present cardiac arrhythmias, atrial fibrillation and osteoporosis and the health problems of aged patients, especially those with cardiovascular disease or other co-morbidity, should be carefully evaluated to enable prescription of an individualized thyroxine dose.
3. The evaluation of the actual metabolic significance and implications of a low TSH value should be made taking into consideration four parameters: a) the relation between $\mathrm{TSH}$ and serum free $\mathrm{T} 3$ and $\mathrm{T} 4, \mathrm{~b}$ ) the qualities of thyroxine preparations, c) the TSH normal range for each individual and, most important, d) the values of serum FT4 and FT3 that accompany a suppressed TSH.

The negative feedback action of free T4 on pituitary TSH is logarithmic, which means that small changes of serum free T4 do not have an analogous small impact on TSH and may provoke quantitatively important changes. Moreover, the activity of pituitary deiodinase, which regulates TSH suppression by transforming T4 to T3, presents individual variations and is subject to many influences, including T4 itself, that can lead to hyperfunctioning or hypofunctioning states with analogous consequences on TSH suppression. Small changes in serum T4 levels due to difference in absorption and metabolism of T4 or other causes can easily occur in exogenous SubHyper and have no or insignificant metabolic consequences but can exert a suppressive action on TSH disproportional to their magnitude.

The variable potency and bioavailability of thyroxine preparations are well known and have a serious bearing on therapeutic choice that aims at avoiding over- or under-dosage. ${ }^{32-34}$ Considerable differences in potency and stability of thyroxine were reported by Hennessey who found superpotency in 9 and subpotency in 47 of 58 batches of thyroxine preparations. ${ }^{35}$ Olveira et al have shown that simple changes in the manufacture of levothyroxine tablets may produce important variations in their bioavailability. ${ }^{36}$ These differences in potency and the potential but undefined contamination of thyroxine preparations with T3 together with the undetermined stability of these compounds, their variable dissolution in biologic fluids and their variable absorption create uncertainty about their biological efficacy that can only be resolved by empirically testing it in each particular case. ${ }^{37}$

The diagnosis of exogenous SubHyper is based on the acceptance of a population based reference range for serum TSH values. However, Andersen et $\mathrm{al}^{38}$ evaluated 15 normal men who were studied 
monthly for a year and demonstrated much more narrow individual fluctuations than the population reference range and a great variation between subjects. This means that an individual may present with a TSH value outside of his "personal" TSH normal range and consequently abnormal for this individual but normal in line with the population reference range. On the other hand, the great variations of $\mathrm{TSH}$ and T4 between subjects indicate a different sensitivity to T4 and TSH interaction among individuals, which explains the variable effect of thyroxine administration in different people.

4. Finally, attention should be paid to an issue that has been given little consideration, namely the FT4 and FT3 values that accompany a suppressed TSH value, since FT4 and FT3 generate and reflect the thyroid metabolic state of the organism. So far, pertinent conclusions are based on the assumption that a low TSH is the result of an excess of free thyroid hormones and that the degree of TSH suppression is an indirect index of the magnitude of this excess and its consequences. However, because of the very nature of serum FT3 and FT4 interactions with TSH, a certain degree of TSH suppression does not necessarily correlate with serum FT3 and FT4 values. This lack of reciprocal relation between TSH and FT3 and FT4, caused by variable individual sensitivity, instability of thyroxine preparations and bioavailability, is well illustrated in the study of Carr et al. In this study of 21 hypothyroid patients, it was shown that by increasing the thyroxine dose that maintained the TSH within the normal reference range by $25 \mu \mathrm{g}$, the TSH value dropped to below $0.2 \mathrm{mIU} / \mathrm{L}$ in most subjects, and with a $50 \mu \mathrm{g}$ overdosage, suppressed TSH value was achieved in all patients. ${ }^{39}$ The free T3 value, however, remained within the normal reference range (3-8 pmol/L) in all but 2 subjects who received the plus $25 \mu \mathrm{g}$ and 3 in the plus $50 \mu \mathrm{g}$ dose. Seventy-five percent of the values ranged from 5 to $6.5 \mathrm{pmol} / \mathrm{L}$ in the plus $25 \mu \mathrm{g}$ dosage and more than half of the values ranged between 5.5 and $7 \mathrm{pmol} / \mathrm{L}$ in the $+50 \mu \mathrm{g}$ dosage. Due to this non-reciprocity between TSH and FT4 and FT3, it is not rare to come across patients receiving $100 \mu \mathrm{g}$ T4 and having suppressed TSH, while others with similar features maintain TSH levels of $2.5 \mathrm{mIU} /$

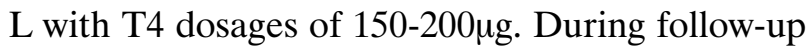

in both cases, it is not unusual to observe a reversal of the situation to either direction. In endogenous SubHyper, a proportion of patients with low TSH values present with normal values at follow-up, leading some authors to advocate as initial strategy surveillance rather than treatment. ${ }^{8,40}$ The question, therefore, arises as to which parameter should be taken to indicate tissue thyrotoxicity and consequent harm to the organism: the degree of TSH suppression or the corresponding levels of FT3 and FT4? Until this issue is satisfactorily scientifically addressed, the practicing physician is justified in feeling that FT3 and FT4 concentrations around the middle of the normal reference range are fairly reassuring of a normal thyroid metabolic state in spite of the presence of a low TSH which may remain suppressed long after reducing the dose of thyroxine. This seems to be the position of 185 members of the American Thyroid Association who were asked to express their opinion on the management of 4 hypothetical cases of different ages with endogenous Hyper presenting T3 and T4 values in the middle of normal range and TSH $0.2-0.3 \mathrm{mIU} / \mathrm{L}$ or undetectable. It was obvious that the clinical judgment of the thyroid specialists is based on the normal T3 and T4 rather than the suppressed or even undetectable TSH. With regard to exogenous SubHyper, the decision for its management will be based on a balanced appraisal of the following issues:

a. The risks and benefits of maintaining suppressive doses of thyroxine or adjusting the dosage;

b. The need to categorize the patients up to the point of individualization;

c. The instability of the efficacy of thyroxine preparations;

d. The actual thyroid metabolic state reflected by serum FT4 and FT3 concentrations.

The issue of exogenous SubHyper cannot adequately be addressed by an evidence-based methodology or specific guidelines because of the lack of definitive data and primarily because of the multitude of variations that each individual presents. The task, therefore, of the practicing endocrinologist is to scrutinize each patient's data and characteristics and employ his best clinical judgment with the aim 
of applying an individualized optimal therapeutic scheme to his patient. It must be stressed, however, that TSH suppressive therapy is essential in the management of patients with high risk differentiated thyroid cancer. ${ }^{42}$

\section{REFERENCES}

1. Hollowell JG, Staehling NW, Flanders WD, et al, 2002 Serum TSH, T4 and thyroid antibodies in the United States population (1988 to 1994): National Health and Nutrition Examination Survey (NHANES III). J Clin Endocrinol Metab 87: 489-499.

2. Surks MI, Ortiz E, Daniels GH, et al, 2004 Subclinical thyroid disease: scientific review and guidelines for diagnosis and management. JAMA 291: 228-238.

3. Michalopoulou G, Alevizaki M, Piperingos G, et al, 1998 High serum cholesterol levels in persons with "high-normal" TSH levels: should one extend the definition of subclinical hypothyroidism? Eur J Endocrinol 138: 141-145.

4. Wartofsky L, Dickey RA, 2005 The evidence for a narrower thyrotropin reference range is compelling. J Clin Endocrinol Metab 90: 5483-5488.

5. Surks MI, Goswami G, Daniels GH, 2005 The thyrotropin reference range should remain unchanged. J Clin Endocrinol Metab 90: 5489-5496.

6. Vanderpump MP, Tunbrdge WM, French JM, et al, 1995 The incidence of thyroid disorders in the community: a twenty-year follow-up of the Whickham Survey. Clin Endocrinol (Oxf) 43: 55-68.

7. Canaris GJ, Manowitz NR, Mayor G, Ridgway EC, 2000 The Colorado Thyroid Disease Prevalence Study. Arch Intern Med 160: 526-534.

8. Parle JV, Franklyn JA, Cross KW, Jones SC, Sheppard MC, 1991 Prevalence and follow-up of abnormal thyrotrophin $(\mathrm{TSH})$ concentrations in the elderly in the United Kingdom. Clin Endocrinol (Oxf) 34: 7783.

9. Cooper DS, Ridgway EC, 2002 Thoughts on prevention of thyroid disease in the United States. Thyroid 12: 925-929.

10. Brander A, Viikinkoski P, Nickels I, Kivissari L, 1991 Thyroid gland: screening in a random adult population. Radiology 181: 683-687.

11. Mazzaferri EL, 1993 Management of a solitary thyroid nodule. N Engl J Med 28: 553-559.

12. Reiners CR, Wegscheider K, Schicha H, et al, 2004 Prevalence of thyroid disorders in the working population of Germany: Ultrasonography screening in 96,278 unselected employees. Thyroid 14: 926-932.

13. Bonnema SJ, Bennedbaek FN, Wiersinga WM, Hegedós L, 2000 Management of the nontoxic multinodular goitre: A European questionnaire study. Clin Endocrinol 53: 5-12.
14. Helfand M, 2004 Screening for subclinical thyroid dysfunction in nonpregnant adults: A summary of the evidence for the U.S. preventive services task force. Ann Intern Med 140: 128-141.

15. Koutras DA, 1999 Subclinical hyperthryoidism. Thyroid 9: 311-315.

16. Toft AD, 2001 Clinical Practice. Sublinical hyperthyroidism N Eng J Med 345: 512-516.

17. Kahaly GJ, Dillmann WH, 2005 Thyroid action on the heart. Endocr Reviews 26: 704-728.

18. Gharib H, Tuttle RM, Baskin HJ, et al, 2005 Consensus Statement \#1: Subclinical thyroid dysfunction: a joint statement on management from the American Association of Clinical Endocrinologists, the American Thyroid Association, and the Endocrine Society. Thyroid 15: 24-28.

19. Biondi B, Palmieri EA, Klein M, Schumberger M, Filetti S, Lambardi G, 2005 Subclinical hyperthryroidism: clinical features and treatment options. Eur J Endocrinol 152: 1-9.

20. Papi G, Pearce EN, Braverman LE, Betterle C, Roti E, 2005 A clinical and therapeutic approach to thyrotoxicosis with thyroid-stimulating hormone suppression only. Am J Med 118: 349-361.

21. Parle JV, Maisonneuve P, Sheppard MC, Boyle P, Franklyn JA, 2001 Prediction of all-cause and cardiovascular mortality in elderly people from one low serum thyrotropin result: a 10-year cohort study. Lancet 358: 861-865.

22. Letters to the Editor 2002 Lancet 359: 797-800.

23. Sawin CT, Geller A, Wolf PA, et al, 1994 Low serum thyrotropin concentrations as a risk factor for atrial fibrillation in older persons. N Engl J Med 331: 12491252.

24. Auer J, Scheiber P, Mische T, Langsteger W, Eber O, Eber B, 2001 Subclinical hyperthyroidism as a risk factor for atrial fibrillation. Am Heart J 142: 838-842.

25. Smit JWA, Eustatia-Rutten CFA, Corssmit EPM, et al, 2005 Reversible diastolic dysfunction after long-term exogenous subclinical hyperthyroidism: a randomized, placebo, controlled study. J Clin Endocrinol Metab 90: 6041-6047.

26. Faber J, Galloe AM, 1994 Changes in bone mass during prolonged subclinical hyperthyroidism due to Lthyroxine treatment: a meta-analysis. Eur J Endocrinol 130: 350-356.

27. Uzzan B, Campos J, Cucherat M, Nony P, Boissel JP, Perret GY, 1996 Effects on bone mass of long-term treatment with thyroid hormones: a meta-analysis. J Clin Endocrinol Metab 81: 4278-4289.

28. Franklin JA, Betteridge J, Daykin J, et al, 2002 Longterm thyroxine treatment and bone mineral density. Lancet 340: 9-13.

29. Bauer DC, Ettinger B, Nevitt M, Stone KL, 2001 The Study of Osteoporotic Fractures Research Group. Risk for fracture in women with low serum levels of thy- 
roid-stimulating hormone. Ann Intern Med 134: 561568.

30. Vestergaard P, Mosekilde L, 2002 Fractures in patients with hyperthyroidism and hypothyroidism: a nationwide follow-up study in 16,249 patients. Thyroid 12: 411-419.

31. Biondi B, Fazio S, Carella C, et al, 1994 Control of adrenergic over-activity by b-blockade improves quality of life in patients receiving long-term suppressive therapy with levothyroxine. J Clin Endocrinol Metab 78: 1028-1033.

32. Stockigt J, 2004 Testing the bioavailability of oral Lthyroixine by studying its absorption: smoke or mirrors? Thyroid 14: 167-168.

33. Klein I, Danzi S, 2003 Evaluation of the therapeutic efficacy of different levothyroxine preparations in the treatment of human thyroid disease. Thyroid 13: 11271132.

34. Blakesley V, Awni W, Locke C, Ludden T, Granneman GR, Braverman LE, 2004 Are bioequivalence studies of levothyroxine sodium formulations in euthyroid volunteers reliable? Thyroid 14: 191-200.

35. Hennesey JV, 2003 Levothyroxine a new drug? Since when? How could that be? Thyroid 13: 279-282.

36. Olveira G, Almaraz MC, Soriguer F, et al, 1997 Altered bioavailability due to changes in the formulation of commercial preparations of levothyroxine in patients with differentiated thyroid carcinoma. Clin Endocrinol (Oxf) 46: 707-711.

37. Koutras DA, 2003 The treacherous use of thyroxine preparations. Stability of thyroxine preparations. Hormones 2: 161-168.

38. Andersen S, Pedersen KM, Bruun NH, Lauberg P, 2002 Narrow individual variations in serum T4 and T3 in normal subjects: a clue to the understanding of subclinical hypothyroidism. J Clin Endocrinol Metab 87: 1068-1072.

39. Carr D, Mcleod DT, Parry G, Thomes HM, 1988 Fine adjustment of thyroxine replacement dosage: comparison of the thyrotrophin releasing hormone test using a sensitive thyrotopin assay with measurement of free thyroid hormones and clinical assessment. Clin Endocrinol (Oxf) 28: 325-333.

40. Woeber KA, 2005 Observations concerning the natural history of subclinical hyperthyroidism. Thyroid 15 : 687.

41. McDermott MT, Woodmansee WW, Haugen BR, Smart A, Ridgway C 2003 The management of subclinical hyperthyroidism by thyroid specialists. Thyroid 13: $1133-1139$.

42. Biondi B, Filetti S, Schlumberger M, 2005 Thyroidhormone therapy and thyroid cancer: a reassessment. Nat Clin Pract Endo Metabol 1: 32-40. 